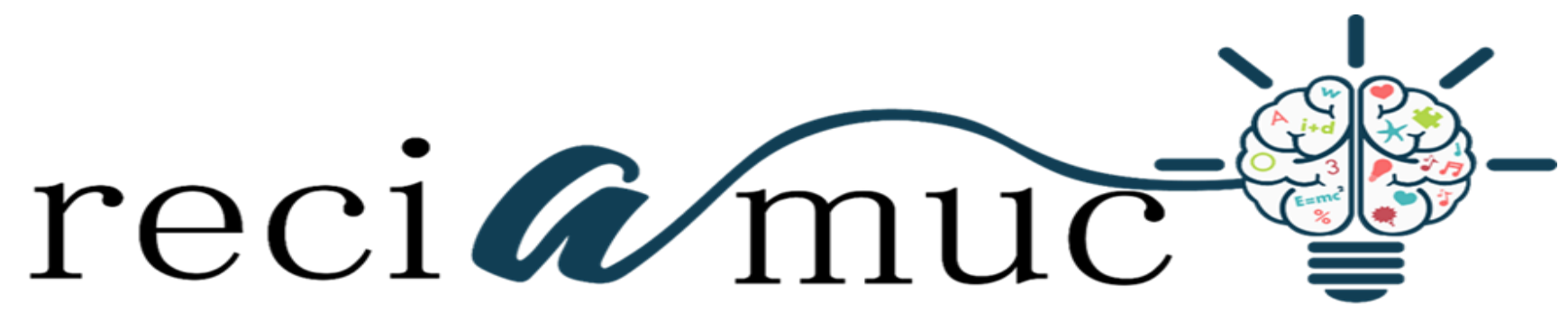

Revista cientifica de investigación actualización del mundo de las ciencias

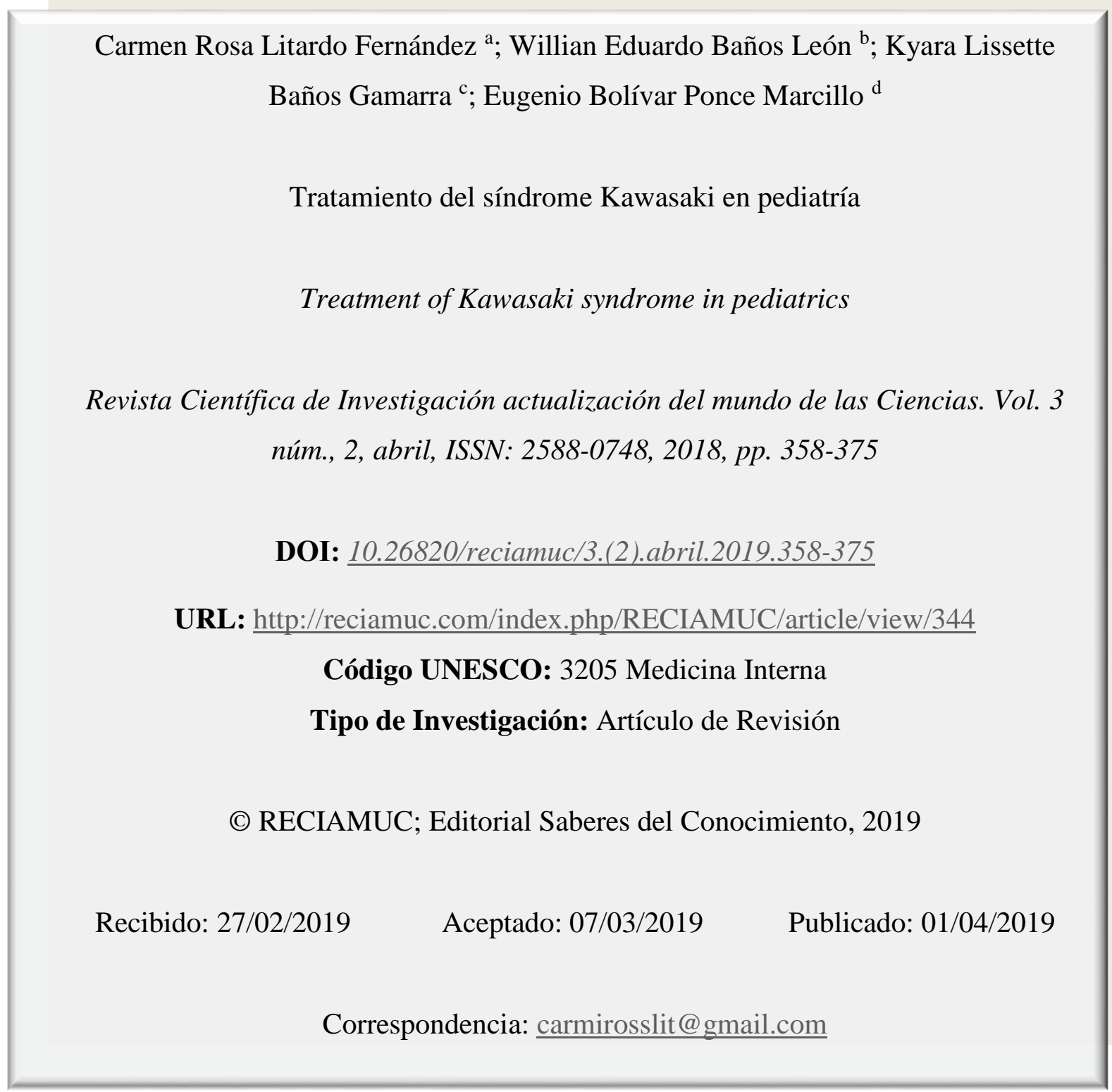

a. Médico; Investigador Independiente; Guayaquil, Ecuador; carmirosslit@gmail.com

b. Médico; Investigador Independiente; Guayaquil, Ecuador; willianb_88@ hotmail.com

c. Médico; Investigador Independiente; Guayaquil, Ecuador; kyabanosq@ gmail.com

d. Médico; Investigador Independiente; Guayaquil, Ecuador; samuelaurelio2010@ @otmail.com 


\section{Tratamiento del síndrome Kawasaki en pediatría}

Vol. 3, núm. 2., (2019)

Carmen Rosa Litardo Fernández; Willian Eduardo Baños León; Kyara Lissette Baños Gamarra; Eugenio Bolívar Ponce Marcillo

\section{RESUMEN}

La principal causa de enfermedad cardiaca adquirida en niños en países en vías de desarrollo es el síndrome de Kawasaki. Aproximadamente un 25\% de los pacientes llegan desarrollar aneurismas coronarios por no recibir tratamiento, sin embargo, los que lo reciben de manera temprana, hacen que esa proporción tienda a disminuir en 5\%. Mediante la propuesta de un diseño documental, con la presente revisión se delimita como objetivo principal la realización de un resumen actualizado referente al tratamiento del síndrome de Kawasaki, considerando para ello la necesidad de conceptualizarla y a la vez describir los aspectos más resaltantes tales como los etiológicos, patológicos y genéticos, al igual que su diagnóstico, a fines de poner a disposición un contenido bibliográfico vigente sobre esta patología, útil tanto para el médico tratante como para cualquier otro interesado en conocer sobre dicha enfermedad y su tratamiento. Se concluye resaltando la importancia de un diagnóstico certero y oportuno, que en definitiva es lo trascendental a la hora de establecer el tratamiento idóneo en fase aguda, a fines de disminuir al mínimo posible la incidencia de alteraciones coronarias y a su vez, las consecuencias a largo plazo que se presentan.

Palabras claves: Síndrome; Aneurismas; Etiológicos; Patológicos; Fase. 


\title{
Tratamiento del síndrome Kawasaki en pediatría
}

Vol. 3, núm. 2., (2019)

Carmen Rosa Litardo Fernández; Willian Eduardo Baños León; Kyara Lissette Baños Gamarra; Eugenio Bolívar Ponce Marcillo

\begin{abstract}
The main cause of acquired heart disease in children in developing countries is Kawasaki syndrome. Approximately $25 \%$ of patients develop coronary aneurysms for not receiving treatment, however, those who receive it early, cause that proportion tends to decrease by $5 \%$. By means of the proposal of a documentary design, with the present revision the main objective is defined to carry out an updated summary referring to the treatment of Kawasaki syndrome, considering for this the need to conceptualize it and at the same time describe the most outstanding aspects such as the etiological ones, pathological and genetic, as well as its diagnosis, in order to make available a current bibliographic content on this pathology, useful both for the attending physician and for any other interested in knowing about said disease and its treatment. It is concluded by highlighting the importance of an accurate and timely diagnosis, which is ultimately the transcendental when establishing the right treatment in the acute phase, in order to minimize the incidence of coronary alterations and, in turn, the long-term consequences term presented.
\end{abstract}

Key words: Syndrome; Aneurysms; Etiological; Pathological; Phase. 


\section{Tratamiento del síndrome Kawasaki en pediatría}

Vol. 3, núm. 2., (2019)

Carmen Rosa Litardo Fernández; Willian Eduardo Baños León; Kyara Lissette Baños Gamarra;

Eugenio Bolívar Ponce Marcillo

\section{Introducción.}

Molina, Barquero, \& Peña (2019) inician su trabajo investigativo refiriendo que, por primera vez, ésta enfermedad fue descrita en 1964 por el Dr.Tamisaku Kawasaki, como un síndrome mucocutánea linfático, ya que hasta ese momento la afectación cardiaca de la enfermedad asociada no había sido determinada. Posteriormente, en 1970, por una parte, se empezó a ver que de forma imprevista morían los pacientes con este síndrome por oclusiones trombóticas de las arterias coronarias, y por otra, el Dr.Noboru Tanaka logra hallar en autopsias de niños que padecían dicha enfermedad, evidencias de aneurismas y trombosis coronarias. De la misma manera afirman que, hasta ahora, se desconoce la etiología de esta enfermedad que, mayormente, afecta a infantes menores de 5 años, no obstante, puede presentarse a cualquier edad, condición que es conocida como síndrome atípico o incompleto de Kawasaki. (Molina, Barquero, \& Peña; 2019; p. 36)

Por otra parte De Pedro (2018), mediante su investigación ha llegado a asegurar que la enfermedad de Kawasaki (EK) “es la causa más común de enfermedad cardiaca adquirida en niños en países desarrollados y la segunda causa de vasculitis en la infancia”, agregando que la mayoría de los casos (85\%) ocurre en menores de 5 años, y su mayor incidencia se refleja entre los 18 y 24 meses de edad. Agregan que, aunque hoy en día "no se ha identificado un desencadenante único", las referencias de otros estudios han sugerido que es posible que su origen o desencadenante sea infeccioso, esto es, igualmente a la par de otras teorías recientes que aluden a una posible toxina fúngica. Para el diagnóstico, se considera expedita y fundamentalmente, la evaluación conjunta de 


\section{Tratamiento del síndrome Kawasaki en pediatría}

Vol. 3, núm. 2., (2019)

Carmen Rosa Litardo Fernández; Willian Eduardo Baños León; Kyara Lissette Baños Gamarra; Eugenio Bolívar Ponce Marcillo

notaciones de manejo práctico, indicadores inflamatorios en las pruebas de laboratorio y criterios clínicos. (De Pedro, 2018)

Santos et. al. (2018) manejan datos epidemiológicos muy parecidos a los antes mencionados, no obstante, destacan que dicha enfermedad "Por lo general se presenta más en varones que en mujeres con una razón 1.5:1 en promedio” (p. 17)

Con esta revisión sistemática se estima realizar un resumen general actualizado referente a esta enfermedad, describiendo sus aspectos etiológicos, patológicos y genéticos, diagnóstico y tratamiento, a fines de poner a disposición un contenido bibliográfico actualizado sobre esta patología, útil tanto para el médico tratante como para cualquier otro interesado en conocer sobre dicha enfermedad y su tratamiento, siendo todo ello en aras de evitar las complicaciones a largo plazo.

\section{Materiales y Métodos.}

Las herramientas y materiales de trabajo que se conciben para el desarrollo de este trabajo investigativo son los siguientes: computadores personales con conexión a internet y un cúmulo de contenidos científico-académicos diversos, con los que se persigue aportar información de calidad y actualizada respecto al Tratamiento del síndrome de Kawasaki en pediatría. Se escoge un diseño documental, de tipo no experimental, a nivel descriptivo, de corte transeccional y bajo una metodología inductiva.

Esta investigación se enfoca en la búsqueda y revisión sistemática de literatura científico académica seleccionada, que por una parte, está disponible determinadas bases de datos, entre las 


\section{Tratamiento del síndrome Kawasaki en pediatría}

Vol. 3, núm. 2., (2019)

Carmen Rosa Litardo Fernández; Willian Eduardo Baños León; Kyara Lissette Baños Gamarra; Eugenio Bolívar Ponce Marcillo

que figuran: MedlinePlus, PubMed, Biblioteca Virtual de la Salud (BVS), SciELO, Dialnet y ELSEVIER, Cochrane, entre otras; y por la otra, mediante el uso del material físicamente disponible en la biblioteca universitaria como en otras particulares, representando todo ello parte esencial del proceso investigativo y de comprensión que facilita la síntesis de la mejor evidencia disponible, y a su vez representando todo ello uno de los resultados esperados.

Principalmente se realiza una búsqueda aleatoria y consecutiva en las mencionadas bases de datos, usando las expresiones "síndrome de kawasaki”, “enfermedad de kawasaki”, “tratamiento del kawasaki” y “kawasaki”, lo que aproximadamente resultó en más de un centenar de miles de registros bibliográficos. Luego éstos se filtran en base a criterios de: idioma español, relevancia, correlación temática y fecha de publicación en los últimos siete años, sin descartar por tipo de material bibliográfico; es decir, se escogen títulos de artículos científicos, ensayos, revisiones sistemáticas, protocolos, editoriales, libros, boletines, folletos, tesis de grado, posgrado y doctorado, noticias científicas, entre otros documentos e información de interés científico y académico.

Seguidamente, se efectúa la selección y clasificación de la literatura científico académica físicamente disponible, aplicando, en términos generales, los criterios antes mencionados, siendo a partir de allí que el equipo investigador le da la correspondiente lectura crítica y análisis de toda esa evidencia científica, lo que resultó consecutiva y consensuadamente en el fundamento de las ideas y planteamientos aquí plasmados. 


\section{Tratamiento del síndrome Kawasaki en pediatría}

Vol. 3, núm. 2., (2019)

Carmen Rosa Litardo Fernández; Willian Eduardo Baños León; Kyara Lissette Baños Gamarra; Eugenio Bolívar Ponce Marcillo

\section{Resultados.}

Aspectos etiológicos, patogénicos y genéticos

Santos et. al. (2018) en su obra dicen que, a pesar de que hay evidencia de datos clínicos y epidemiológicos que se inclinan a la concepción de una etiología infecciosa, básicamente viral, sigue siendo en base a otras fuentes que se puede asegurar e indicar que la etiología de esta enfermedad es aún desconocida. Igualmente se fundamenta en el aporte de otros tratadistas para expresar que "Los cambios patológicos presentes son el resultado de una respuesta inmunitaria exagerada y patológica a un agente en las células endoteliales de sujetos genéticamente predispuestos" (p. 17-18)

En el Hospital Infantil de Stanford (2019) actualmente se coincide con la misma idea antes expuesta, ya que al respecto indican que:

No está claro qué causa la enfermedad de Kawasaki. Los científicos creen que un virus o una bacteria pueden ser los responsables, pero aún hay muchas investigaciones en curso actualmente. La enfermedad de Kawasaki no parece ser contagiosa, ni tampoco hereditaria. (Stanford Children's Health, 2019)

De una manera más completa Barrios et. al. (2018) explica que, independientemente de que el origen o desencadenante infeccioso sea sugerido por hallazgos clínicos, de laboratorio y las características epidemiológicas de la enfermedad, en la actualidad "no se ha podido identificar un agente etiológico infeccioso único" ni tampoco existe evidencia de "que la enfermedad se asocie a la exposición a fármacos, o que se desarrolle en respuesta a un superantígeno”. (p. 188. e3) 


\section{Tratamiento del síndrome Kawasaki en pediatría}

Vol. 3, núm. 2., (2019)

Carmen Rosa Litardo Fernández; Willian Eduardo Baños León; Kyara Lissette Baños Gamarra; Eugenio Bolívar Ponce Marcillo

Al respecto, continúan explicando que, entre las diversas tesis que hoy día justifican el origen de dicha enfermedad, mayormente figura la que sugiere que "puede ser causada por un agente infeccioso que se inhalaría e infectaría células epiteliales bronquiales ciliadas de tamaño mediano". Así mismo, indican que existen estudios recientes que se basan "en el análisis de las grandes epidemias de EK en Japón" en los que sus resultados apuntan a que pudiera estarse tratando de "un agente medioambiental transportado por vientos troposféricos, posiblemente una toxina fúngica". A la par de todo esto, también resalta el factor genético del huésped, y eso es debido a "la alta incidencia en las comunidades asiáticas y el mayor riesgo entre hermanos de los casos".

Se han publicado algunos estudios de asociación de genoma completo (GWAS) en EK11 y se han identificado varios loci biológicamente implicados en la inflamación, la respuesta inmunitaria y el estado cardiovascular. Así, una hipótesis razonable mente abierta, es que la EK está causada por un agente infeccioso aún por identificar, que produce enfermedad solo en individuos genéticamente predispuestos, particularmente asiáticos. Su rareza en los primeros meses de vida y en adultos sugiere un agente al que estos últimos son inmunes y del cual los lactantes muy pequeños están protegidos por anticuerpos maternos pasivos. (Barrios et. al.; 2018; p. 188. e3)

\section{Diagnóstico.}

De conformidad a la idea expuesta por especialistas tales como Gil, Nieto, León, \& Hernández (2018), en esta oportunidad se logra comprender y conocer que, a pesar de que a lo largo del tiempo los criterios diagnósticos considerados para la detección del síndrome de 


\section{Tratamiento del síndrome Kawasaki en pediatría}

Vol. 3, núm. 2., (2019)

Carmen Rosa Litardo Fernández; Willian Eduardo Baños León; Kyara Lissette Baños Gamarra; Eugenio Bolívar Ponce Marcillo

Kawasaki han sido constantemente modificados, en la actualidad los que se usan con más frecuencia son los de: A) la "Guía del Comité de Investigación de la enfermedad de Kawasaki” (Guías Japonesas 2002); en las que, para un diagnóstico inicial, indican que deben suceder al menos cinco de los siguientes seis criterios: fiebre persistente mayor de cinco días, congestión conjuntival bilateral, cambios en labios y cavidad oral, exantema polimorfo, cambios en región distal de extremidades, y/o linfodenopatía cervical no purulenta; y B) la "Guía para el diagnóstico de la enfermedad de Kawasaki de la Sociedad Americana del Corazón" (American Heart Association (AHA)), en la que se indica debe considerarse: $1^{\circ}$ ) una fiebre que persiste al menos cinco días con, $2^{\circ}$ ) al menos cuatro de las cinco características clínicas principales, que serían: i) cambios en extremidades (Agudo: eritema de palmas, plantas; edema de manos, pies / subagudo: descamación periungueal de manos y pies entre la semana dos y tres); ii) exantema polimorfo (máculo-papular difuso, urticarial, eritrodermia, eritema multiforme, no vesicular o buloso); iii) inyección conjuntival bilateral no exudativa; iv) cambios en labios y cavidad oral: eritema, labios agrietados, lengua en fresa, inyección difusa en mucosa oral y faríngea; v) linfadenopatía cervical (mayor de $1.5 \mathrm{~cm}$ de diámetro), generalmente unilateral; $3^{\circ}$ ) exclusión de otras enfermedades con hallazgos similares (escarlatina, infecciones virales como sarampión, adenovirus, síndrome de Stevens-Johnson, síndrome de shock tóxico)

Además, resaltan que, aunque existen otros datos clínicos que no se incluyen o se consideran como criterios en dichas guías, vale la pena mencionarlos por lo clave que se consideran en un diagnóstico inicial. Los signos clínicos referidos son: la descamación perineal, que aparece, por lo general, unos días antes de la descamación de extremidades, y la reactivación del sitio de 


\section{Tratamiento del síndrome Kawasaki en pediatría}

Vol. 3, núm. 2., (2019)

Carmen Rosa Litardo Fernández; Willian Eduardo Baños León; Kyara Lissette Baños Gamarra; Eugenio Bolívar Ponce Marcillo

inyección de la vacuna BCG (bacilo Calmette-Guérin), que se observa con mayor frecuencia que la linfadenopatía cervical. (Gil, Nieto, León, \& Hernández; 2018; p. 154-155)

\section{Tratamiento.}

Entre las recomendaciones que ofrece el Instituto del corazón de Texas de los Estados Unidos de América, destaca una alta dosificación (administración) de aspirina ya que ello coadyuva en la reducción de la formación de "aneurismas, la fiebre, el sarpullido, y la inflamación y dolor articular" aparte de que "puede evitar la formación de coágulos sanguíneos” por el efecto que este medicamento produce en la sangre, que no es otro que el de la dilución de la misma. También enfatizan que la temprana administración intravenosa de gammaglobulina, en ocasiones “puede reducir el riesgo de que se dañen las arterias coronarias".

Explican que, en los casos de niños donde no se evidencia un persistente daño en el corazón o las arterias coronarias, normalmente recupera su estado de salud por completo, no obstante, deben hacerlo evaluar periódicamente por un cardiólogo pediatra, durante aproximadamente un año. Ese mismo proceder es propio en casos de niños con un daño leve en las arterias coronarias, y después de ese periodo, el especialista, posiblemente, recomendará que la consulta disminuya a una frecuencia que pudiera variar de entre 3 y 5 años, con la finalidad de chequear y determinar “qué tanto ejercicio puede hacer sin peligro". Cuando en el niño se evidencia un daño grave en las arterias coronarias, sí es necesaria una consulta periódica con el especialista, aparte de también someterse a estudios de seguimiento, tales como; electrocardiografías o ecocardiografías, hasta los 10 años de edad, aproximadamente. Posterior a eso, quizá se le recomiende disminuir la consulta a una por año y a hacerse una prueba de esfuerzo, a fines de valorar el corazón y determinar 


\section{Tratamiento del síndrome Kawasaki en pediatría}

Vol. 3, núm. 2., (2019)

Carmen Rosa Litardo Fernández; Willian Eduardo Baños León; Kyara Lissette Baños Gamarra; Eugenio Bolívar Ponce Marcillo

igualmente qué tanto ejercicio pueden hacer sin peligro. Para el tratamiento de los efectos a largo plazo de esta enfermedad, es posible realizar intervenciones quirúrgicas o coronarias percutáneas. (Texas Heart Institute, s.f.)

Las indicaciones referidas por otra respetable institución son las de Mayo Clinic (2016) asegurando que, en base a que los objetivos del tratamiento inicial de éste enfermedad son los de bajar la fiebre y la inflamación, y prevenir el daño cardíaco; el médico tratante debe procurar reducir el riesgo de complicaciones, por lo que debe comenzar el tratamiento en el niño lo antes posible, claro está, después de la aparición de los signos y síntomas, preponderantemente, cuando aún presente fiebre.

Para cumplir estos objetivos, indican que el especialista debe referir:

- Gammaglobulina. Debido a que la infusión de una proteína inmune (gammaglobulina) a través de una vena (intravenosa) puede disminuir el riesgo de problemas de la arteria coronaria.

- Aspirina. Ya que las dosis altas de aspirina pueden ayudar a tratar la inflamación, aliviar el dolor y la inflamación articular, reducir la fiebre. El tratamiento de Kawasaki es una excepción rara a la norma de no administrar aspirina a los niños, pero sólo si se la administra bajo la supervisión del médico tratante.

Debido a los riesgos de complicaciones graves, el tratamiento inicial para la enfermedad de Kawasaki generalmente se administra en un hospital. 


\section{Tratamiento del síndrome Kawasaki en pediatría}

Vol. 3, núm. 2., (2019)

Carmen Rosa Litardo Fernández; Willian Eduardo Baños León; Kyara Lissette Baños Gamarra; Eugenio Bolívar Ponce Marcillo

Después del tratamiento inicial, verificando que el paciente no padece más fiebre, es posible que se necesite indicar la toma de una dosis baja de aspirina durante al menos seis semanas o más si contrae un aneurisma en la arteria coronaria, debido a que ello ayuda a prevenir la formación de coágulos. Esta administración deberá suspenderse en caso de que el niño contraiga gripe o varicela, mientras dure el tratamiento de éstas, y esto es porque existen evidencia que relaciona la toma de aspirina con el síndrome de Reye, una afección poco frecuente pero potencialmente mortal que puede afectar la sangre, el hígado y el cerebro de los niños y los adolescentes después de una infección viral.

Cabe destacar que, en promedio, la enfermedad de Kawasaki dura 12 días si no es tratada, pero las complicaciones cardíacas pueden manifestarse después y durar más tiempo. Con tratamiento, el niño puede comenzar a mejorar rápidamente después del primer tratamiento con gammaglobulina.

Si el paciente tiene alguna indicación de problemas cardíacos, es probable que se le recomiende hacerse unas pruebas de seguimiento para supervisar la salud del corazón en intervalos regulares, esto es indicado para hacerse, por lo general, a las seis u ocho semanas después de haber comenzado la enfermedad, para luego repetirlas seis semanas después. Si el caso de problemas cardíacos es persistente, es posible una derivación a un cardiólogo pediátrico.

En casos muy particulares, un niño con un aneurisma de la arteria coronaria podría necesitar lo siguiente: 


\section{Tratamiento del síndrome Kawasaki en pediatría}

Vol. 3, núm. 2., (2019)

Carmen Rosa Litardo Fernández; Willian Eduardo Baños León; Kyara Lissette Baños Gamarra; Eugenio Bolívar Ponce Marcillo

- Medicamentos anticoagulantes. Estos medicamentos, como la aspirina, el clopidogrel (Plavix), la warfarina (Coumadin, Jantoven) y la heparina, ayudan a prevenir la formación de coágulos.

- Angioplastia de la arteria coronaria. Este procedimiento abre las arterias que tienen un estrechamiento tal que impide el flujo sanguíneo al corazón.

- Colocación de stent. Este procedimiento consiste en implantar un dispositivo en la arteria obstruida para ayudar a mantenerla abierta y disminuir las posibilidades de otro bloqueo. La colocación de un stent suele acompañar a la angioplastia.

- Injerto de bypass en la arteria coronaria. Esta operación consiste en redirigir la sangre alrededor de una arteria coronaria enferma a través del injerto de una porción de vaso sanguíneo de la pierna, del pecho o del brazo, a fin de usarlo como ruta alternativa.

- Esperar a la vacunación

Destacan que, si el paciente estuvo bajo la administración de gammaglobulina, es recomendable esperar por lo menos 11 meses para aplicar las vacunas de la varicela o del sarampión, porque la gammaglobulina puede afectar la eficiencia de estas vacunas. (Mayo Clinic, 2016)

Para De Pedro (2018), quien contextualiza su aporte en base al trabajo realizado por varios especialistas representantes del Grupo de Cardiología Clínica de la Sociedad Española de Cardiología Pediátrica y Cardiopatías Congénitas (SECPCC); la administración medicamentosa de inmunoglobulina intravenosa a la par del ácido acetil salicílico, sigue siendo "el tratamiento de primera línea". Precisa que, el uso clásico del referido ácido en conjunto con la inmunoglobulina, 


\section{Tratamiento del síndrome Kawasaki en pediatría}

Vol. 3, núm. 2., (2019)

Carmen Rosa Litardo Fernández; Willian Eduardo Baños León; Kyara Lissette Baños Gamarra; Eugenio Bolívar Ponce Marcillo

se debe a la eficacia que en estudios previos se ha evidenciado de éste como coadyuvante, independientemente de que, al parecer, el mismo no disminuya la frecuencia de aparición de aneurismas coronarios. Informa que, a pesar de este tratamiento entre un 10-20\% de los enfermos tienen fiebre, siendo particularmente sobre estos casos cuando toca discutir sobre la terapia con corticoides (concebidos como terapia preventiva en EK grave y de rescate para los que no responden inicialmente), fármacos biológicos u otros inmunosupresores, pues al parecer "podrían suprimir la inflamación vascular". Alega que hay estudios hechos con Infliximab, y también con ciclosporina, que han demostrado la disminución de la fiebre y el tiempo de hospitalización pero no las consecuencias cardiacas. Con la especialista alega que "existen 3 casos publicados con mejoría de secuelas cardiacas a corto plazo, disminuyendo los días de fiebre y los parámetros analíticos". Este consenso no sólo es una guía de apoyo en esos pacientes "difíciles" tanto por enfermedad grave en un inicio, como por falta de respuesta al tratamiento inicial. También marca de forma sencilla los controles a los que se deberían someter los niños con enfermedad de Kawasaki pasada según el tipo y evolución de sus secuelas coronarias. Los pacientes adultos con antecedentes de enfermedad de Kawasaki necesitan una transición adecuada desde el cardiólogo infantil al de adultos. (De Pedro, 2018)

De la investigación de Escobar et. al. (2019), quienes la desarrollaron en base al estudio y análisis comparativo de 51 fuentes informativas, 24 de ellas relevantes en cuanto al tratamiento farmacológico del síndrome de Kawasaki, pudo entenderse que éstos se han basado en el argumento de otros tratadistas para indicar que, de acuerdo a la mejor evidencia disponible, el tratamiento a seguir en cualquiera de los casos de esta enfermedad, se inclina al de primera línea “[...] consistente en la administración de terapia combinada de ASA más inmunoglobulina G, el 


\section{Tratamiento del síndrome Kawasaki en pediatría}

Vol. 3, núm. 2., (2019)

Carmen Rosa Litardo Fernández; Willian Eduardo Baños León; Kyara Lissette Baños Gamarra; Eugenio Bolívar Ponce Marcillo

cual tiene efecto comprobado sobre la disminución de la inflamación y la reducción de la incidencia de aneurismas coronarios". De la misma manera han referido que:

Evidencia de primer nivel sugiere que la adición de corticoesteroides al tratamiento convencional parece ser segura, pues disminuye la tasa de fallo y retratamiento con inmunoglobulina G y la incidencia de anomalías coronarias; sin embargo, algunos estudios han mostrado limitaciones metodológicas que impiden generalizar su uso, quedando indicados a criterio clínico en los casos con escasa respuesta o refractarios a inmunoglobulina, en enfermedad severa y ante la presencia de anomalías ecocardiográficas tempranas, sin que exista una pauta de tratamiento bien definida. (p. 106) En esta parte, se considera importante referir el hallazgo de un respetable estudio de revisión elaborado por Wardle, Connolly, Seager, \& Tulloh (2017), quienes precisamente analizaron la repercusión por el uso de un conjunto de fármacos conocidos como corticoesteroides en niños afectados por EK "para la reducción de las perspectivas de problemas cardíacos a futuro, así como el efecto sobre la duración de la fiebre, los signos de infección en la sangre y el número de días en el hospital". Los especialistas aclaran que, en su estudio de revisión sistemática, "las pruebas están actualizadas hasta noviembre 2016" y que incluyeron a "niños y niñas con diagnóstico de enfermedad de Kawasaki”. Así mismo indican que seleccionaron siete “ensayos clínicos con asignación aleatoria” en el que figura la participación de 922 pacientes, siendo en dichos ensayos que se "compararon la administración de corticosteroides con el no uso de corticosteroides. Finalmente, llama poderosamente la atención que éstos expertos concluyen su estudio asegurando que: 


\section{Tratamiento del síndrome Kawasaki en pediatría}

Vol. 3, núm. 2., (2019)

Carmen Rosa Litardo Fernández; Willian Eduardo Baños León; Kyara Lissette Baños Gamarra; Eugenio Bolívar Ponce Marcillo

Las pruebas de calidad moderada indican que la administración de corticosteroides en la fase aguda de la EK puede asociarse con una mejoría en las anomalías de la arteria coronaria, una duración más corta de la estancia hospitalaria y una menor duración de los síntomas clínicos. Las pruebas de alta calidad muestran una reducción de los niveles de marcadores inflamatorios. [...] Las pruebas presentadas en este estudio indican que el tratamiento con un ciclo prolongado de corticosteroides debe considerarse para todos los niños con diagnóstico de EK hasta que se realicen más estudios. (Wardle, Connolly, Seager, \& Tulloh, 2017)

Volviendo a la obra de Escobar (2019), respecto a los casos resistentes a la inmunoglobulina, éstos autores han referido que la alternativa de tratamiento es la indicación del infliximab (anticuerpo monoclonal de la inmunoglobulina G1, específico contra el FNT- $\alpha 1$, que produce una rápida resolución del cuadro agudo, sin embargo, ello no quiere decir que se recomiende su uso pues su eficacia aún no ha sido comprobada, y eso mismo ha pasado con los estudios sobre los demás fármacos inmunosupresores utilizados en otros tipos de vasculitis.

En definitiva, estos tratadistas llegan a concluir que:

El pilar de tratamiento de la enfermedad de Kawasaki es la administración de ASA más inmunoglobulina G. La evidencia, aunque limitada, parece orientarse en favor de los corticoesteroides para el tratamiento de formas severas, refractarias a inmunoglobulina $\mathrm{G}$ y de rápida progresión. Las demás alterativas, incluyendo los antagonistas del factor de necrosis tumoral alfa y otras terapias inmunosupresoras, se reservan para casos sin respuesta a tratamiento convencional, sin que exista evidencia en favor de su uso en términos de seguridad y eficacia. (Escobar et al.; 2019; p. 107) 


\section{Tratamiento del síndrome Kawasaki en pediatría}

Vol. 3, núm. 2., (2019)

Carmen Rosa Litardo Fernández; Willian Eduardo Baños León; Kyara Lissette Baños Gamarra; Eugenio Bolívar Ponce Marcillo

\section{Conclusiones.}

Es sumamente importante que, ante cualquier caso de manejo clínico de un estado febril a nivel pediátrico, primero que todo, se deben poseer amplios e inequívocos conocimientos respecto a este tipo de enfermedades, aspecto delicado sobre todo por tratarse la salud en niños, y además, dentro de las consideraciones asumidas en cuanto al diagnóstico diferencial, mantener siempre presente esta clase de patologías (que pudiera en determinado momento verse complicado su diagnóstico por las razones etiológicas ya expuestas), y eso es, independientemente de que a la par se estén presentando otros virus respiratorios; pues se cree así es que puede lograrse un diagnóstico oportuno que en definitiva es lo trascendental a la hora de establecer el tratamiento idóneo en el lapso de los primeros diez días desde el comienzo de la fiebre (fase aguda), y todo ello es con la intención de disminuir al mínimo posible (aproximadamente a un 5\%) la incidencia de alteraciones coronarias (fundamentalmente la formación de aneurismas coronarios) y a su vez, las consecuencias a largo plazo que se presentan.

\section{Bibliografía}

Barrios, A., Centeno, F., Rojo, H., Fernández, E., Sánchez, ,., \& Pérez, J. (Septiembre de 2018). Consenso nacional sobre diagnóstico, tratamiento y seguimiento cardiológico de la enfermedad de Kawasaki. Anales de Pediatría, 89(3), 188.e1-188.e22. doi:10.1016/j.anpedi.2018.04.003

De Pedro, S. (21 de noviembre de 2018). epeap.org. Recuperado el 20 de 07 de 2019, de Sociedad Española de Pediatría Extrahospitalaria y Atención Primaria: https://sepeap.org/manejode-la-enfermedad-de-kawasaki-consenso-nacional/

Escobar, H., Meneses, G., Ijají, J., Triana, H., Molina, J., Vidal, J., . . . Cedeño, A. (2019). Tratamiento farmacológico de la enfermedad de Kawasaki. (F. Escobar Córdoba, \& J. Eslava Schmalbac, Edits.) Revista de la Facultad de Medicina de la Universidad Nacional de Colombia, 67(1), 103-108. doi:https://doi.org/10.15446/revfacmed.v67n1.64144 


\section{Tratamiento del síndrome Kawasaki en pediatría}

Vol. 3, núm. 2., (2019)

Carmen Rosa Litardo Fernández; Willian Eduardo Baños León; Kyara Lissette Baños Gamarra;

Eugenio Bolívar Ponce Marcillo

Gil, G., Nieto, N., León, A., \& Hernández, Z. (2018). https://www.medigraphic.com/ pdfs/juarez/ju-2018/ju183f.pdf. Revista del Hospital Juan de Dios de México, 58(3), 154158. Recuperado el 15 de 07 de 2019, de https://www.medigraphic.com/pdfs/juarez/ju2018/ju183f.pdf

Mayo Clinic. (26 de Octubre de 2016). mayoclinic.org. Recuperado el 15 de 07 de 2019, de mayoclinic.org: https://www.mayoclinic.org/es-es/diseases-conditions/kawasaki-disease/ diagnosis-treatment/drc-20354603

Molina, V., Barquero, D., \& Peña, M. (Enero de 2019). Impacto de la enfermedad de Kawasaki en la población pediátrica. Revista Médica Sinergia, 4(1), 35-45. doi:https://doi.org /10.31434/rms.v4i1.201

Santos, L., Reyes, K., Reyes, U., Guerrero, M., López, G., Quero, A., de Lara, J. (Enero-Marzo de 2018). Enfermedad de Kawasaki: Conceptos actuales. Avan C Salud Med, 5(1), 17-26. Recuperado el 15 de 07 de 2019, de http://www.salud.oaxaca.gob.mx/wp-content/ uploads/2018/07/Acs-181-03r.pdf

Stanford Children's Health. (2019). stanfordchildrens.org. Recuperado el 15 de julio de 2019, de stanfordchildrens.org: https://www.stanfordchildrens.org/es/topic/default?id=enfermedad dekawasaki-90-P04904

Texas Heart Institute. (s.f.). texasheart.org. Recuperado el 15 de 07 de 2019, de texasheart.org: https://www.texasheart.org/heart-health/heart-information-center/topics/enfermedad-dekawasaki/

Wardle, A., Connolly, G., Seager, M., \& Tulloh, R. (27 de Enero de 2017). Corticosteroids for the treatment of Kawasaki disease in children. (I. John Wiley \& Sons, Ed.) Cochrane Database of Systematic Reviews, 1(CD011188), 1-52. doi:10.1002/14651858.CD011188.pub2

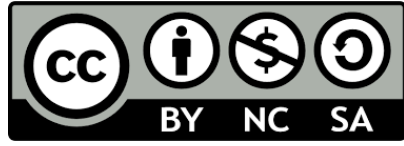

RECONOCIMIENTO-NOCOMERCIAL-COMPARTIRIGUAL

CC BY-NC-SA

ESTA LICENCIA PERMITE A OTROS ENTREMEZCLAR, AJUSTAR Y CONSTRUIR A PARTIR DE SU OBRA CON FINES NO

COMERCIALES, SIEMPRE Y CUANDO LE RECONOZCAN LA AUTORÍA Y SUS NUEVAS CREACIONES ESTÉN BAJO UNA LICENCIA CON LOS MISMOS TÉRMINOS. 